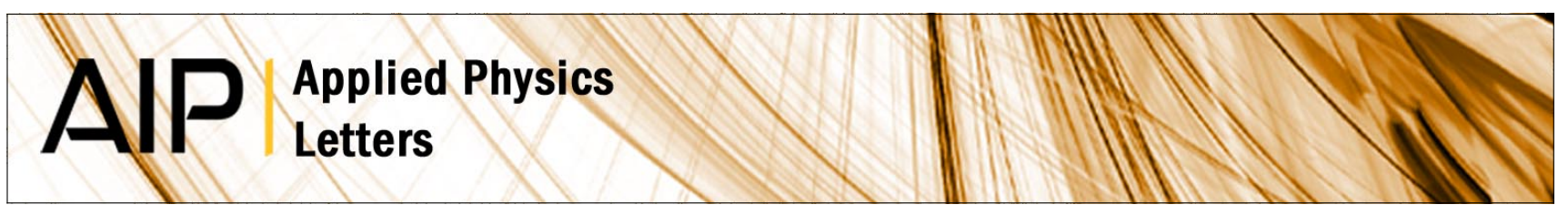

\title{
Anomalous thermal relaxation in carbon nanoclusters
}

Alexander V. Savin and Yuri S. Kivshar

Citation: Appl. Phys. Lett. 98, 193106 (2011); doi: 10.1063/1.3590256

View online: http://dx.doi.org/10.1063/1.3590256

View Table of Contents: http://apl.aip.org/resource/1/APPLAB/v98/i19

Published by the American Institute of Physics.

\section{Related Articles}

High temperature thermal properties of thin tantalum nitride films Appl. Phys. Lett. 99, 261906 (2011)

Ballistic phonon thermal transport in a cylindrical semiconductor nanowire modulated with nanocavity J. Appl. Phys. 110, 124321 (2011)

Synthesis of zinc fulleride (ZnxC60) thin films with ultra-low thermal conductivity J. Appl. Phys. 110, 124320 (2011)

Interfacial thermal resistance between metallic carbon nanotube and $\mathrm{Cu}$ substrate J. Appl. Phys. 110, 124314 (2011)

Multiscale modeling of cross-linked epoxy nanocomposites to characterize the effect of particle size on thermal conductivity

J. Appl. Phys. 110, 124302 (2011)

\section{Additional information on Appl. Phys. Lett.}

Journal Homepage: http://apl.aip.org/

Journal Information: http://apl.aip.org/about/about_the_journal

Top downloads: http://apl.aip.org/features/most_downloaded

Information for Authors: http://apl.aip.org/authors

\section{ADVERTISEMENT}

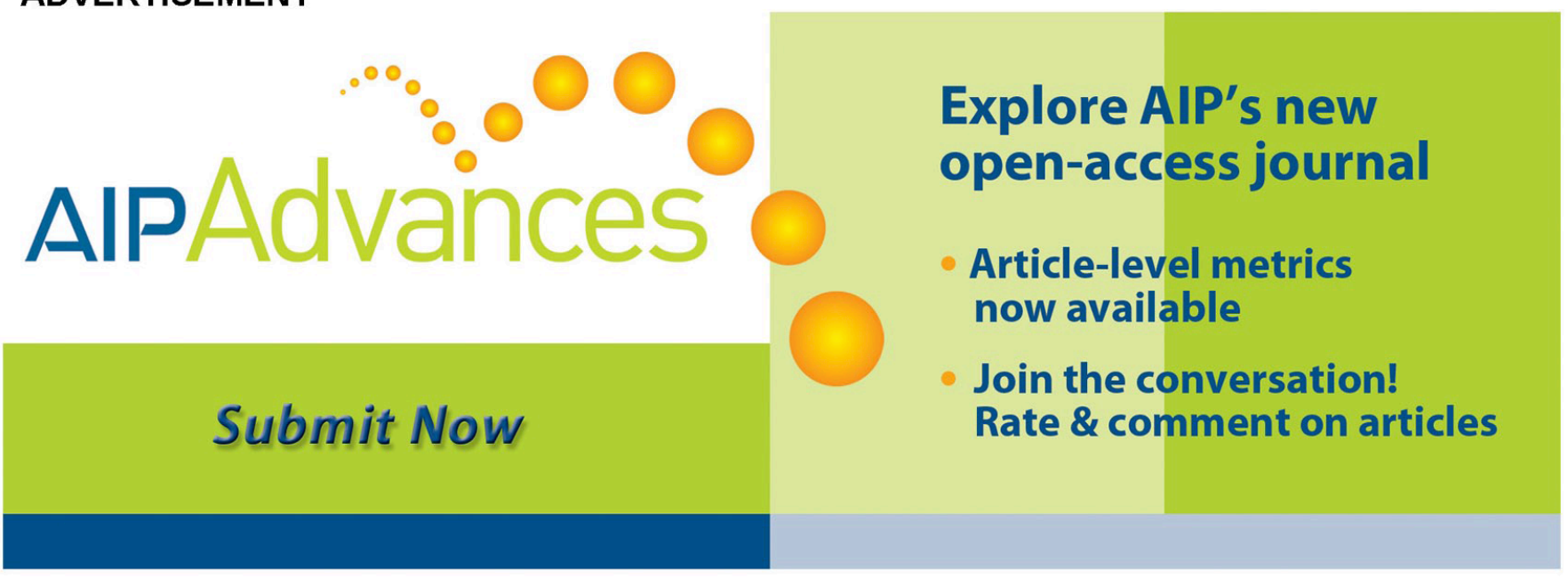




\title{
Anomalous thermal relaxation in carbon nanoclusters
}

\author{
Alexander V. Savin ${ }^{1,2, a)}$ and Yuri S. Kivshar ${ }^{1}$ \\ ${ }^{1}$ Nonlinear Physics Center, Research School of Physics and Engineering, Australian National University, \\ Canberra, Australian Capital Territory 0200, Australia \\ ${ }^{2}$ Semenov Institute of Chemical Physics, Russian Academy of Sciences, Moscow 119991, Russia
}

(Received 11 February 2011; accepted 20 April 2011; published online 11 May 2011)

\begin{abstract}
By employing molecular dynamics simulations, we study thermal relaxation in two types of nanoclusters, a cubelike stack of identical carbon nanotubes and a nanocube of fullerene $\mathrm{C}_{60}$ molecules. We calculate relaxation time and coefficient of thermal conductivity and reveal that the $\mathrm{C}_{60}$ nanocluster demonstrates anomalously slow thermal relaxation due to the excitation of long-lived internal localized oscillatory modes. This suggests that such nanoclusters would be the most efficient for photophoretic manipulation of fullerenes. (c) 2011 American Institute of Physics. [doi:10.1063/1.3590256]
\end{abstract}

When a surface of an absorbing particle is heated nonuniformly, the surrounding gas molecules rebound off the surface with different velocities creating an integrated force on the particle. This phenomenon is called thermophoresis, a mass transfer induced by the temperature gradient. The specific form of this phenomenon is associated with light heating being known as photophoresis. The thermal forces are not negligible when the particles are small and under large temperature gradients, and such forces depend of the particle morphology. ${ }^{1}$

Recently, a novel principle of optical trapping and manipulation was demonstrated to increase substantially the manipulation distance by means of strong thermal forces ${ }^{2}$ (see also Refs. 3 and 4). The experimental demonstrations employed two types of absorbing particles, (i) carbon nanoclusters produced by laser ablation and (ii) hollow glass carbon-coated microspheres.

Being inspired by the experimental observations that the thermal forces and trapping efficiency depend strongly on the structure and morphology of carbon clusters, in this letter, we study how the thermal relaxation of the carbon nanoclusters and the resulting thermal force depend on their specific morphology and internal structure.

To analyze the effect of morphology on the heat relaxation and thermal conductivity, We consider two types of cubic nanoclusters having the same shape but different internal structure, as shown in Figs. 1(a) and 1(b). The first nanocluster is created by a stack of $12 \times 10$ identical armchair $(6,6)$ carbon nanotubes $\mathrm{C}_{1104}$, as shown in Fig. 1(a). The other cluster is a nanocube of $12 \times 12 \times 10$ fullerene $\mathrm{C}_{60}$ molecules (buckyballs C60-Ih), see Fig. 1(b).

We model the intermolecular interaction in carbon nanotubes $\mathrm{C}_{1104}$ and molecules $\mathrm{C}_{60}$ with the help of the Brenner potentials. ${ }^{5,6}$ Then in the ground state the nanotube with the chirality index $(6,6)$ and consisting of 1104 carbon atoms has the length $L_{x}=11.417 \mathrm{~nm}$ and the radius $R=0.418 \mathrm{~nm}$, whereas a spherical molecule $\mathrm{C}_{60}$ has the radius $R$ $=0.363 \mathrm{~nm}$.

We describe the interaction of carbon atoms from different molecules by means of the Lennard-Jones 12-6 potential $U_{\mathrm{LJ}}(r)=4 \epsilon_{\mathrm{LJ}}\left[(\sigma / r)^{12}-(\sigma / r)^{6}\right]$, where $r$ is the distance be-

${ }^{a)}$ Electronic mail: asavin@ center.chph.ras.ru. tween the centers of atoms, energy $\epsilon_{\mathrm{LJ}}=0.004556 \mathrm{eV}$, and parameter $\sigma=0.3851 \mathrm{~nm}$. Then, the cubic nanocluster composed of $12 \times 10$ nanotubes $\mathrm{C}_{1104}$ has the following dimensions: $L_{x}=11.417 \mathrm{~nm}, L_{y}=11 d_{y}+2 R=12.144 \mathrm{~nm}, L_{z}$ $=9.5 d z+2 R=12.075 \mathrm{~nm}$, where the period along the axis $y$ is $d_{y}=1.028 \mathrm{~nm}$, and along the axis $z, d_{z}=1.183 \mathrm{~nm}$, see Fig. 1(a). The cubic structure of $12 \times 12 \times 10$ fullerene molecules $\mathrm{C}_{60}$ has the following dimensions: $L_{x}=11 d_{x}+2 R$ $=10.11 \mathrm{~nm}, \quad L_{y}=11 d_{y}+2 R=10.68 \mathrm{~nm}, \quad L_{z}=9.5 d_{z}+2 R$ $=10.65 \mathrm{~nm}$, and the period along the axis $x d_{x}=0.853 \mathrm{~nm}$, the axis $y d_{y}=0.905 \mathrm{~nm}$, and the axis $z d_{z}=1.045 \mathrm{~nm}$-see Fig. 1(b).

To study thermal conductivity and heat relaxation we employ the Langevin equation

$$
M \ddot{\mathbf{u}}_{\alpha, i}=-\partial H / \partial u_{\alpha, i}-\Gamma M \dot{\mathbf{u}}_{\alpha, i}+\Xi_{\alpha, i},
$$

where $M=19.92 \times 10^{-27} \mathrm{~kg}$ is the mass of carbon atom, the three-dimensional (3D) vector $\mathbf{u}_{\alpha, i}$ defines the position of the $i$ atom of the molecule with the number $\alpha$ [for nanotubes $\alpha=\left(n_{y}, n_{z}\right), n_{y}=1, \ldots, 12, n_{z}=1, \ldots, 10$, and for fullerene molecules $\mathrm{C}_{60}$ the index $\alpha=\left(n_{x}, n_{y}, n_{z}\right), n_{x}=1, \ldots, 12, n_{y}$ $=1, \ldots, 12$, and $\left.n_{z}=1, \ldots, 10\right], H$ is the system Hamiltonian, $\Gamma=1 / t_{r}$ is the Langevin collision frequency with $t_{r}$ being the corresponding particle relaxation time, and $\Xi_{\alpha, i}=\left\{\xi_{\alpha, i, j}\right\}_{j=1}^{3}$ is $3 \mathrm{D}$ vector of Gaussian distributed stochastic forces with correlation functions

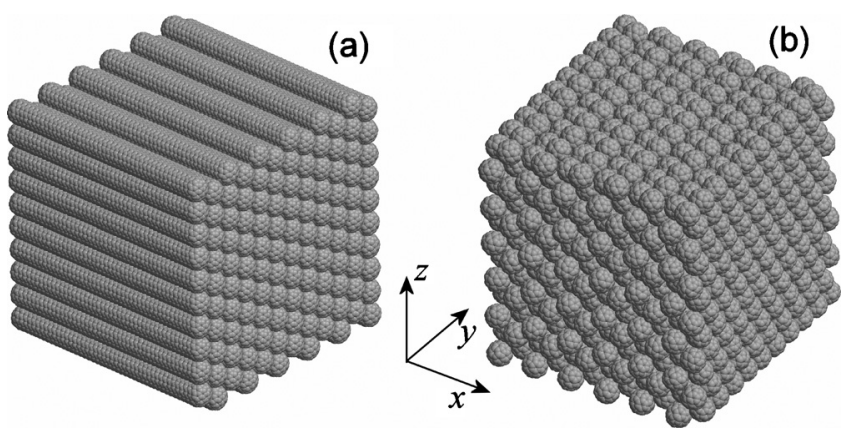

FIG. 1. Structure of two cubic carbon nanoclusters: (a) a stack of $12 \times 10$ identical armchair $(6,6)$ carbon nanotubes $\mathrm{C}_{1104}$ (with length $L_{x}$ $=11.417 \mathrm{~nm}$, and lattice spacings $d_{y}=1.028 \mathrm{~nm}$ and $\left.d_{z}=1.183 \mathrm{~nm}\right)$ and (b) nanocube created by $12 \times 12 \times 10$ fullerenes $\mathrm{C}_{60}$ molecules (with the lattice spacings $d_{x}=0.853 \mathrm{~nm}, d_{y}=0.905 \mathrm{~nm}$, and $\left.d_{z}=1.045 \mathrm{~nm}\right)$. 


$$
\left\langle\xi_{\alpha, i, j}\left(t_{1}\right) \xi_{\beta, k, l}\left(t_{2}\right)\right\rangle=2 M \Gamma k_{B} T_{\alpha, i} \delta_{\alpha \beta} \delta_{i k} \delta_{j l} \delta\left(t_{2}-t_{1}\right),
$$

$k_{B}$ is Boltzmann constant, $T_{\alpha, i}$ temperature of the molecule $(\alpha, i)$.

We model the thermal relaxation of a cubelike nanocluster when one of its sides is heated to a higher temperature than the rest of it, see Fig. 1. The thermal force, such as thermophoretic or photophoretic force, is defined by the temperature difference between the opposite sides of the nanocluster. This force will be more effective for slower thermal relaxation.

Nanoclusters composed of carbon nanotubes represent highly anisotropic structures where different forces are responsible for the bonding in the longitudinal and transverse directions. We consider the thermal relaxation of the nanocluster when all surface atoms at one of the sides are heated to the temperature $T_{1}=500 \mathrm{~K}$, whereas all other atoms have the temperature $T_{0}=300 \mathrm{~K}$. To achieve such a thermalized state, we solve the Langevin Eq. (1) with the initial conditions corresponding to the ground state of the nanocluster during the time $t=20 t_{r}$ (where $t_{r}$ is the relaxation time which for the systems under consideration can be take as $t_{r}$ $=0.1 \mathrm{ps}$ ). For thermalization of the side $x z$ in the Langevin equation we take $T_{\alpha, i}=T_{1}$ for the atoms of the first surface layer of the nanotubes perpendicular to the axis $y$ [see Fig. 1(a)], and for the thermalization of the side $y z$ we should take the temperature $T_{\alpha, i}=T_{1}$ for all atoms at the right surface hemispheres of the nanotubes [see Fig. 4(a)]. For all other atoms we take $T_{\alpha, i}=T_{0}$.

As a result of such thermalization procedure, it appears a temperature difference $\Delta T=T_{1}-T_{0}$ between the opposite sides of the nanocluster. First, we study the relaxation of nanoclusters without their interaction with the Langevin thermostat, this correspond to omission of the last two terms in Eq. (1).

Nanoclusters created by spherical fullerene molecules $\mathrm{C}_{60}$ are isotropic structures. Therefore, we may study only the thermal relaxation when the temperature gradient applied, say, along the axis $x$. To create a thermalized state with the help of the Langevin equation, we take temperature $T_{\alpha, i}$ $=T_{1}$ for all molecules which belong to the surface layer of the cluster [see Fig. 1(b)], and for all other molecules we put $T_{\alpha, i}=T_{0}$.

Our numerical simulations demonstrate that both types of nanoclusters are stable to thermal fluctuations and their shape does not change substantially. Dependence of the temperature difference $\Delta T$ versus time $t$ for the nanoclusters composed of identical carbon nanotubes is shown in Fig. 2. As follows from these results the temperature gradient decays exponentially, $\Delta T \sim \exp \left(-t / t_{0}\right)$, when $t \rightarrow \infty$. However, the thermal relaxation along the axis $x$ goes 50 times faster than that along the axis $y$, with the relaxation time $t_{0}$ $=3.3 \mathrm{ps}$ and $t_{0}=166 \mathrm{ps}$, respectively.

Evolution of the temperature difference $\Delta T$ at two opposite sides of the nanocluster composed of the fullerene molecules $C_{60}$ is shown in Fig. 3. As follows from Fig. 3, the temperature gradient decays much slower in this case, and it follows a power law, $\Delta T \sim t^{-1}$. It is important to notice that if we treat the $C_{60}$ molecules as rigid atoms and do not take into account their internal dynamics, then $\Delta T$ decays much faster and in accord with the exponential law, $\Delta T$ $\sim \exp \left(-t / t_{0}\right)$ with the characteristic time $t_{0}=4.4 \mathrm{ps}$.

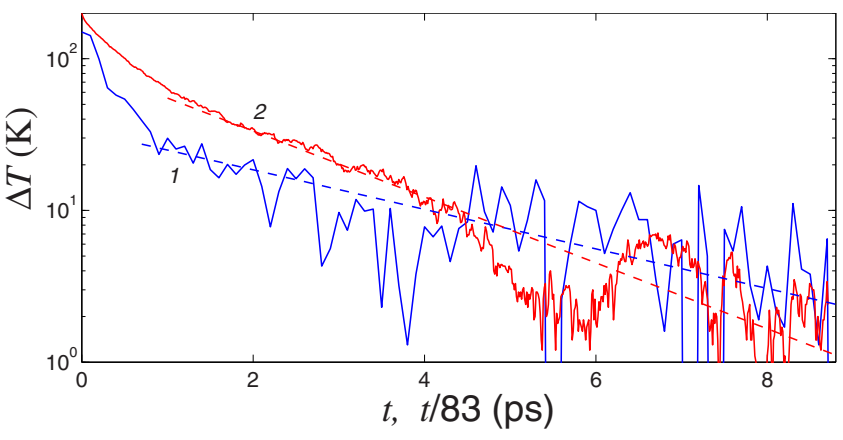

FIG. 2. (Color online) Temporal evolution of the temperature difference $\Delta T$ between the opposite sides of the nanocluster composed of carbon nanotubes where the temperature gradient is applied along the axis $x$ (curve 1) and along the axis $y$ (curve 2, reduced time scale $t / 83$ is applied). Dashed lines mark the exponential dependence $\exp \left(-t / t_{0}\right)$ with the relaxation times $t_{0}=3.3 \mathrm{ps}$ and $166 \mathrm{ps}$, respectively.

Therefore, we come to the conclusion that the observed anomalously slow relaxation of the thermal energy in the nanocrystal of $\mathrm{C}_{60}$ fullerene molecules is associated with its internal dynamics. More detailed analysis of the nanocrystal dynamics demonstrates that such structures support longlived localized anharmonic oscillatory modes in the form of discrete breathers with the lifetime exceeding tens of picoseconds. Slow decay of such oscillatory modes explain anomalously slow thermal relaxation in the $\mathrm{C}_{60}$ nanoclusters.

To determine the coefficient of thermal conductivity, we place two opposite sides of the cubic nanocluster into thermostat at different temperatures [see Figs. 4(a) and 4(b)] and find the resulting stationary distribution of the temperature gradient, as shown in Fig. 4(c). To do this, we employ the Langevin Eq. (1) and select the temperature for the molecules at the left surface as $T_{\alpha, i}=T_{+}=330 \mathrm{~K}$ and smaller temperature $T_{\alpha, i}=T_{-}=270 \mathrm{~K}$ for the molecules at the surface of the opposite side, while "switching off" the interaction of all other molecules with the thermostat.

In the absence of thermal exchange between the surfaces and bulk molecules, we should have a balance of the input energy produced by random forces and the energy lost to dissipation. In this case the temperatures at the opposite sides of the nanocluster would be $T_{+}$and $T_{-}$. Thermal exchange between the sides and internal molecules leads to the temperature decrease from the left side and the temperature grows from the right side, $T_{r}: T_{+}>T_{l}>T_{r}>T_{-}$- see Fig.

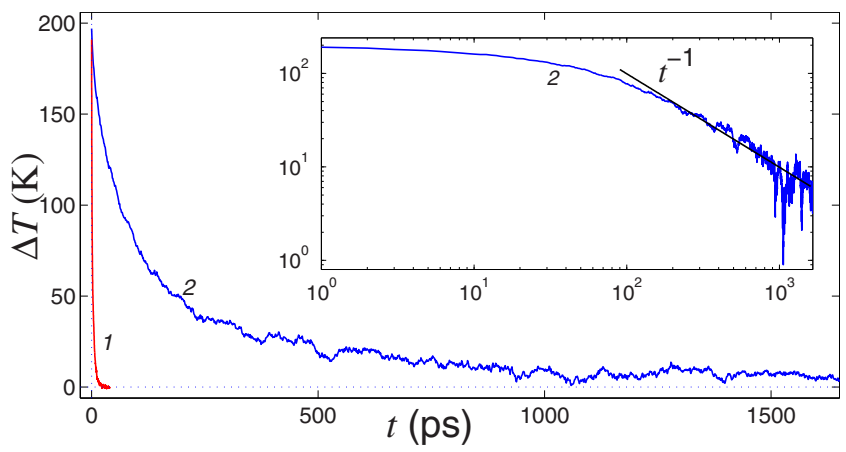

FIG. 3. (Color online) Temporal evolution of the temperature difference $\Delta T$ between the opposite sides of the nanocluster composed of fullerene molecules $\mathrm{C}_{60}$. Curve 1: thermal relaxation of a cluster of rigid molecules, curve 2 : the results of full molecular-dynamic simulations. Inset shows the same dependence (curve 2) in the log scale, where straight line marks the asymptotic dependence $\Delta T \sim t^{-1}$. 

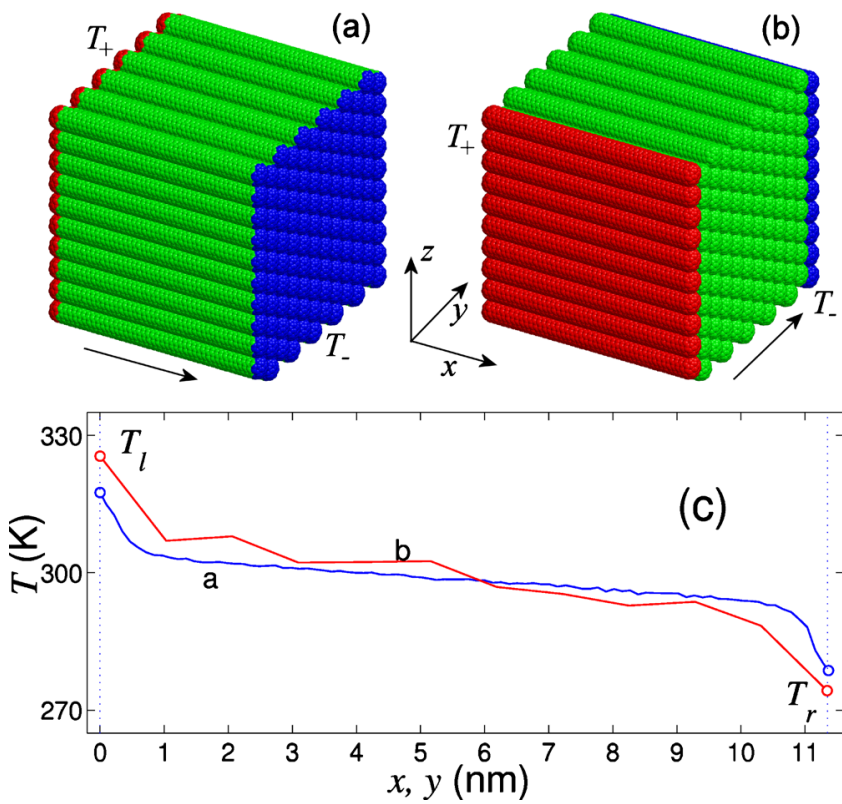

FIG. 4. (Color online) Temperature gradient in a cubic nanocluster composed of a stack of carbon nanotubes when its opposite sides are placed in the Langevin thermostat at the temperatures $T_{+}=330 \mathrm{~K}$ and $T_{-}=270 \mathrm{~K}$; the corresponding "hot" and "cold" atoms are shown by red (grey) and blue (black) colors, respectively. Green (light grey) color show the atoms not interacting with the thermostat. Arrows indicate the direction of the heat flow, (a) along the axis $x$, (b) along the axis $y$. (c) Stationary profile of the temperature gradient. Curve a: along the axis $x$ for the case (a) (relaxation time $t_{r}=0.1 \mathrm{ps}$ ); curve b: along the axis $y$ for the case (b) (relaxation time $t_{r}=10 \mathrm{ps}$ ). Open circles show the stationary temperatures $T_{l}$ and $T_{r}$ at the left and right sides, respectively.

4(c). The energy accumulated in the dissipative forces at the nanocluster surfaces can be defined as $\Gamma M \Sigma\left(\dot{u}_{\alpha, i}, \dot{u}_{\alpha, i}\right)$ $=3 N k_{B} T / t_{r}$, where the summation is carried out for all surface atoms, $N$ is the number of such atoms, $T=T_{l}$ and $T$ $=T_{r}$ are the temperatures of the left and right sides, respectively. The energy flow defined by random forces can be evaluated as $3 N k_{B} T / t_{r}$, where temperature $T=T_{+}$and $T=T_{-}$ for the left and right sides of the nanocluster. Therefore, the energy flow can be determined as $J=N k_{B}\left(T_{+}-T_{l}+T_{r}\right.$ $\left.-T_{-}\right) / 2 t_{r}$, and this formula would allow us to calculate the coefficient of thermal conductivity $\kappa=J L / S\left(T_{l}-T_{r}\right)$, where $L$ is the distance between the opposite sides, and $S$ is the area of one side of the nanocluster. More details for the calculation of the coefficient of thermal conductivity by this method can be found in Ref. 7, where this method was used for the study of thermal conductivity of carbon nanotubes.

Our numerical studies of the thermal energy transfer demonstrate that the nanocluster composed of carbon nanotubes has large thermal conductivity along the nanotubes, $\kappa$ $=57.9 \mathrm{~W} / \mathrm{mK}$, and low thermal conductivity in the transverse direction, $\kappa=0.33 \mathrm{~W} / \mathrm{mK}$. This explains rapid heat relaxation of the temperature gradient along the nanotubes. The nanocluster of fullerene molecules $C_{60}$ demonstrates even lower thermal conductivity, $\kappa=0.101 \mathrm{~W} / \mathrm{mK}$. However, this low value does not explain the observed anomalously slow thermal relaxation. Indeed, if we replace the buckyballs by hard spheres, the coefficient of thermal conductivity will become even smaller, $\kappa=0.095 \mathrm{~W} / \mathrm{mK}$, however, the heat relaxation in this nanocluster will go much faster. This confirms the important role of internal oscillatory modes which give a small contribution to the thermal conductivity but slow down the heat relaxation.

In conclusion, by direct full-scale molecular-dynamics simulations, we have demonstrated that the relaxation of a temperature gradient and heat transfer in carbon nanoclusters depend dramatically on their morphology and specific internal structure. We have discovered anomalously slow thermal relaxation in the nanocrystals composed of fullerene molecules $\mathrm{C}_{60}$, which should result in strong thermal forces most efficient for optical manipulation.

Alexander Savin acknowledges hospitality of the Nonlinear Physics Center at the Australian National University. This work was supported by the Australian Research Council. The authors also thank the Joint Supercomputer Center of the Russian Academy of Sciences for using their computer facilities.

${ }^{1}$ S. Suzuki, K. Kuwana, and R. Dobashi, Int. J. Heat Mass Transfer 52, 4695 (2009).

${ }^{2}$ V. G. Shvedov, A. V. Rode, Y. V. Izdebskaya, A. S. Desyatnikov, W. Krolikowski, and Y. S. Kivshar, Phys. Rev. Lett. 105, 118103 (2010).

${ }^{3}$ V. G. Shvedov, A. S. Desyatnikov, A. V. Rode, W. Krolikowski, and Y. S. Kivshar, Opt. Express 17, 5743 (2009).

${ }^{4}$ A. S. Desyatnikov, V. G. Shvedov, A. V. Rode, W. Krolikowski, and Y. S. Kivshar, Opt. Express 17, 8201 (2009).

${ }^{5}$ D. W. Brenner, O. A. Shenderova, J. A. Harrison, S. J. Stuart, B. Ni, and S. B. Sinnott, J. Phys.: Condens. Matter 14, 783 (2002).

${ }^{6}$ S. Maruyama, Y. Igarashi, Y. Taniguchi, and J. Shiomi, J. Therm. Sci. Technol. 1, 138 (2006).

${ }^{7}$ A. V. Savin, B. Hu, and Y. S. Kivshar, Phys. Rev. B 80, 195423 (2009). 